\title{
Velcro bifurcation in competition models with generalized Holling functional response
}

\author{
M. Farkas, E. Sáez, and I. Szántó
}




\title{
VELCRO BIFURCATION IN COMPETITION MODELS WITH GENERALIZED HOLLING FUNCTIONAL RESPONSE
}

\author{
M. FARKAS, E. SÁEZ, AND I. SZÁNTÓ
}

[Received: January 25, 2005]

\begin{abstract}
A four dimensional system is considered describing the competition of three predator species for a single prey species. The predators functional response is of a generalized Holling III type. Predators begin to grow at the same threshold quantity as prey does. The system has a two dimensional simplex of equilibria which is attracting at low values of the carrying capacity $K$ but gets destabilized as $K$ grows and the predators that are less r-strategists lose ground.
\end{abstract}

Mathematics Subject Classification: 92D25, 34C23

Keywords: velcro bifurcation, competition models, predator-prey systems

\section{INTRODUCTION}

The phenomenon of zip bifurcation was introduced by Farkas [2] in 1984 in conjunction with a system describing the competition of two predator species for a prey. It was assumed that the threshold quantities of prey at which predator growth rate became positive were equal. As a consequence, the system had a one dimensional continuum of equilibria (a "zip"). One of the predators could be identified as a $K$ strategist, the other one as an $r$-strategist. A species is called a $K$-strategist, roughly, if it has a relatively low growth rate and may survive with low carrying capacity $K$. A species is an $r$-strategist if it has a high growth rate (for more see [6]). Clearly, the model was not a structurally stable one, however, it illustrated the intuitively evident fact that at low values of the carrying capacity $K$ both predators might survive but as $K$ grew the $K$-strategist lost ground and only the $r$-strategist survived with the prey. Later a whole class of models were characterized that showed the phenomenon [3], see also [4], and some applications were published, see e.g. [5]. Recently [1] the phenomenon was generalized to a four dimensional system that arose in economypolitology. In this case the system had a two dimensional surface of equilibria that got destabilized as the value of a parameter was increased. We called this a velcro bifurcation. In general, suppose that a system of autonomous differential equations

Supported partially by Hungarian National Foundation for Scientific Research, Grant No. T049464, by USM Grant No. 120121, and FONDECYT Grants No. 1030264, No. 7030005. 
has a two dimensional differentiable manifold of Liapunov stable equilibria and in an interval of a bifurcation parameter this manifold is an attractor of the system. Increasing, say, the bifurcation parameter a curve sweeps through the manifold and the equilibria that are left behind get destabilized, i. e., the part of the manifold that is left behind becomes a repeller while the part yet untouched remains an attractor. In this case we say that the system undergoes a velcro bifurcation.

In the present paper we consider three predator species that compete for a single prey. The functional response of the predators is assumed to be of a generalized Holling III type. The Holling III functional response that is of the form $S^{2} /\left(S^{2}+a^{2}\right)$, where $S$ is the prey quantity, has the property that up to a certain value the growth of $S$ is increasing predator efficiency and, as a consequence, the predator has a stabilizing effect on the population, above this value predator efficiency is decreasing (see May [6]). Here we replace Holling III by $S^{n} /\left(S^{n}+a^{n}\right)$ where $n>1$ is an arbitrary integer. This generalized Holling III functional response preserves the basic properties but is somewhat finer.

In the next Section we introduce the model and establish the conditions under which a velcro bifurcation occurs and in Section 3 we present some computer simulations.

\section{Velcro Bifurcation}

Let us denote the quantity of prey at time $t$ by $S(t)$, the quantity of predator $i$ by $x_{i}(t), i=1,2,3$ and consider the following system that describes the competition of the three predator species for the prey

$$
\begin{aligned}
& \dot{S}=r S(1-S / K)-\sum_{i=1}^{3} m_{i} x_{i} \frac{S^{n}}{a_{i}^{n}+S^{n}}, \\
& \dot{x}_{i}=m_{i} x_{i} \frac{S^{n}}{a_{i}^{n}+S^{n}}-d_{i} x_{i}, \quad i=1,2,3
\end{aligned}
$$

where $r, K, m_{i}, a_{i}, d_{i}>0$ are the intrinsic growth rate of prey, the carrying capacity, the maximal birth rate, the half saturation constant and the death rate of predator $i$, respectively, and $n \geq 2$ is an integer. In absence of predation prey grows up to the value of $K$. If predators are present prey quantity is less. As $S$ tends to infinity, per capita predator birth rate tends to $m_{i}$ (the shape of the predator functional response is a sigmoid one). For the survival of predator $i$ it is , clearly, necessary that the maximal birth rate be larger than the death rate:

$$
m_{i}>d_{i}, \quad i=1,2,3 .
$$

This will be assumed in the sequel. When $S=a_{i}$, then the per capita growth rate is half of the maximal. The lower the half saturation constant $a_{i}$ is, the less prey is needed for the maintenance of the predator. Therefore, we shall consider a predator 
with low half saturation constant a $K$-strategist. We assume that

$$
a_{1}<a_{2}<a_{3} .
$$

Predator $i$ begins to grow only if the right hand side of equation $(2.1)_{i}$ is positive. This is the case if $S>S_{i}:=a_{i} \sqrt[n]{d_{i} /\left(m_{i}-d_{i}\right)}=a_{i} \sqrt[n]{1 /\left(b_{i}-1\right)}$, where $b_{i}=m_{i} / d_{i}>1$. We shall assume that the threshold values of prey are equal for the three predators:

$$
S_{T}:=S_{1}=S_{2}=S_{3} .
$$

Conditions (2.3) and (2.4) imply that

$$
b_{1}<b_{2}<b_{3} .
$$

We shall consider a species with a high ratio of maximal birth rate to death rate as an $r$-strategist. Thus, in our system, predator 1 may be considered a $K$-strategist and predator 3 an $r$-strategist. Predator 2 is in between the two.

Applying the conditions above, we can rewrite our system in the form

$$
\begin{aligned}
& \dot{S}=r S(1-S / K)-\sum_{i=1}^{3} m_{i} x_{i} \frac{S^{n}}{a_{i}^{n}+S^{n}}, \\
& \dot{x}_{i}=\beta_{i} \frac{\left(S^{n}-S_{T}^{n}\right)}{a_{i}^{n}+S^{n}} x_{i}, \quad i=1,2,3,
\end{aligned}
$$

where $\beta_{i}=m_{i}-d_{i}>0$. Clearly, the positive orthant of $\left(S, x_{1}, x_{2}, x_{3}\right)$ space is invariant. We shall consider system (2.6) restricted to $\mathbb{R}_{+}^{4}$.

Lemma 1. System (2.1) and, as a consequence, (2.6) is dissipative, i. e., all solutions are bounded.

Proof. Let us add the four equations of (2.1):

$$
\left(S+x_{1}+x_{2}+x_{3}\right)^{\cdot}=r S(1-S / K)-\sum_{i=1}^{3} d_{i} x_{i} .
$$

If $S>K$ or if $S \leq K$ but $\sum_{i=1}^{3} d_{i} x_{i}>r K$ then this derivative is negative. This means that the trajectories of the system cross the hyperplanes

$$
S+x_{1}+x_{2}+x_{3}=C
$$

from outside to inside if $C$ is sufficiently large. 
The equilibria of system (2.6) are $(0,0,0,0),(K, 0,0,0)$ and the points of the twodimensional plane

$$
\begin{aligned}
E:=\left\{\left(S, x_{1}, x_{2}, x_{3}\right) \in \mathbb{R}_{+}^{4}: S=S_{T}, \sum_{i=1}^{3} \frac{m_{i} S_{T}^{n} x_{i}}{a_{i}^{n}+S_{T}^{n}}=r S_{T}\left(1-S_{T} / K\right)\right. & \\
& \left.\quad x_{i} \geq 0, i=1,2,3\right\}
\end{aligned}
$$

the latter having points in $\mathbb{R}_{+}^{4}$ only if

$$
S_{T}<K
$$

We are to study now the stability of these equilibria. The Jacobian of the system is (summation goes always from 1 to 3 )

$$
J=\left[\begin{array}{cccc}
r(1-2 S / K)-n \sum \frac{m_{i} a_{i}^{n} S^{n-1} x_{i}}{\left(a_{i}^{n}+S^{n}\right)^{2}} & -\frac{m_{1} S^{n}}{a_{1}^{n}+S^{n}} & -\frac{m_{2} S^{n}}{a_{2}^{n}+S^{n}} & -\frac{m_{3} S^{n}}{a_{3}^{n}+S^{n}} \\
n \beta_{1} \frac{S^{n-1}}{\left(a_{1}^{n}+S^{n}\right)} x_{1} & \frac{\beta_{1}\left(S^{n}-S_{T}^{n}\right)}{a_{1}^{n}+S^{n}} & 0 & 0 \\
n \beta_{2} \frac{S^{n-1}}{\left(a_{2}^{n}+S^{n}\right)} x_{2} & 0 & \frac{\beta_{2}\left(S^{n}-S_{T}^{n}\right)}{a_{2}^{n}+S^{n}} & 0 \\
n \beta_{3} \frac{S^{n-1}}{\left(a_{3}^{n}+S^{n}\right)} x_{3} & 0 & 0 & \frac{\beta_{3}\left(S^{n}-S_{T}^{n}\right)}{a_{3}^{n}+S^{n}}
\end{array}\right] .
$$

Substituting $(0,0,0,0)$ we obtain easily that the origin is unstable (a hyperbolic saddle).

Substituting $(K, 0,0,0)$ we obtain similarly that this equilibrium is asymptotically stable if $K<S_{T}$ and it is unstable, a hyperbolic saddle if $K>S_{T}$. The result is in accordance to what has been said before. If the carrying capacity, i. e., the lim sup of prey abundance is less than the threshold value above which predator's quantity may grow, then the predators die out. If (2.7) holds, then the predators may survive. This is precisely the case when the system has equilibria in the interior of the positive orthant. (2.7) will be assumed in the sequel.

We turn now to the stability problem of the points in $E$. Substituting the coordinates of an arbitrary point of $E$ into the Jacobian, a straightforward calculation leads one to the following characteristic polynomial:

$$
\begin{aligned}
D(\lambda)=\lambda^{2}\left(\lambda^{2}+\lambda\left(n S_{T}^{n-1} \sum \frac{m_{i} a_{i}^{n} x_{i}}{\left(a_{i}^{n}+S_{T}^{n}\right)^{2}}-r\left(1-2 S_{T} / K\right)\right)\right. & \\
& \left.+n S_{T}^{2 n-1} \sum \frac{m_{i} \beta_{i} x_{i}}{\left(a_{i}^{n}+S_{T}^{n}\right)^{2}}\right) .
\end{aligned}
$$

This means that each equilibrium point in $E$ has zero as a double eigenvalue and two eigenvalues with real part negative or positive depending on the fact whether the quadratic polynomial in brackets is a stable polynomial or inversely. This polynomial 
is stable iff the coefficient of $\lambda$ is positive, i. e., the equilibrium point in $E$ has two eigenvalues with negative real part if and only if

$$
\sum \frac{m_{i} a_{i}^{n} x_{i}}{\left(a_{i}^{n}+S_{T}^{n}\right)^{2}}>\frac{r}{n S_{T}^{n-1}}\left(1-2 S_{T} / K\right) .
$$

Note that (2.8) holds obviously for each point in $E$ if $S_{T}<K<2 S_{T}$. In what follows $K$ will be considered the bifurcation parameter.

Theorem 1. Under the conditions (2.2) - (2.4), (2.7) if at a point of E (2.8) holds, then this point is stable in the Liapunov sense and a neighbourhood of this point is attractive in the sense that it has a tubular neighbourhood from where it attracts the solutions; if the inequality (2.8) is inverted, then the point is unstable.

Proof. If (2.8) holds, then the equilibrium has a two dimensional centre manifold (clearly the simplex $E$ ) and a two dimensional stable manifold, and by continuity the same holds for each equilibrium in a neighbourhood of the point. The stable manifolds of the equilibria in this neighbourhood fill in a tubular neighbourhood in $\mathbb{R}_{+}^{4}$ (this can be proven analogously to how this was done in [2]), thus, from this tubular neighbourhood every solution tends to some equilibrium in this neighbourhood. The unstability proposition is obvious.

Consider (2.8) with the equality sign:

$$
\sum \frac{m_{i} a_{i}^{n} x_{i}}{\left(a_{i}^{n}+S_{T}^{n}\right)^{2}}=\frac{r}{n S_{T}^{n-1}}\left(1-2 S_{T} / K\right) .
$$

This is the equation of a two dimensional plane in the $S=S_{T}$ section of $\mathbb{R}_{+}^{4}$. If $K$ is small $\left(\leq 2 S_{T}\right)$, then (2.8) holds, obviously, in each point of $E$, i. e., $E$ is an attractor of the system. However, if $K$ is increased, the plane (2.9) may intersect $E$ and, as a consequence, the stability properties of the equilibria in $E$ may change. The following theorem explains what happens.

Theorem 2. Suppose that conditions (2.2) - (2.4) and (2.7) hold and assume that

$$
a_{1}<a_{2}<a_{3}<S_{T} / \sqrt[n]{n-1}
$$

(1) if $K \in\left(S_{T}, \frac{\left((n-2) a_{1}^{n}-2 S_{T}^{n}\right) S_{T}}{(n-1) a_{1}^{n}-S_{T}^{n}}\right)$, then each equilibrium in $E$ is stable in the Liapunov sense and $E$ is an attractor of system (2.6);

(2) if $K \in\left(\frac{\left((n-2) a_{3}^{n}-2 S_{T}^{n}\right) S_{T}}{(n-1) a_{3}^{n}-S_{T}^{n}}, \infty\right)$, then all these equilibria are unstable and $E$ is a repeller;

(3) if $K$ is increased from one end to the other one of the interval

$$
\left(\frac{\left((n-2) a_{1}^{n}-2 S_{T}^{n}\right) S_{T}}{(n-1) a_{1}^{n}-S_{T}^{n}}, \frac{\left((n-2) a_{3}^{n}-2 S_{T}^{n}\right) S_{T}}{(n-1) a_{3}^{n}-S_{T}^{n}}\right),
$$


then the line of intersection of $E$ and the plane (2.9) is traveling through $E$ from the vertex on the axis $x_{1}$ to the vertex on the axis $x_{3}$ and the equilibria left behind get destabilized; for

$$
K \in\left(\frac{\left((n-2) a_{1}^{n}-2 S_{T}^{n}\right) S_{T}}{(n-1) a_{1}^{n}-S_{T}^{n}}, \frac{\left((n-2) a_{3}^{n}-2 S_{T}^{n}\right) S_{T}}{(n-1) a_{3}^{n}-S_{T}^{n}}\right)
$$

this line of intersection divides E into two parts, "the lower one" is a repeller and "the upper one" is an attractor of the system (see Figure 1 in the next Section), i. e., the system undergoes a velcro bifurcation.

Proof. Let us denote the respective coordinate intercepts of $E$ by $\left(x_{E 1}, 0,0\right)$, $\left(0, x_{E 2}, 0\right),\left(0,0, x_{E 3}\right)$ and those of the plane $(2.9)$ by $\left(x_{s 1}, 0,0\right),\left(0, x_{s 2}, 0\right),\left(0,0, x_{s 3}\right)$. A simple calculation yields

$$
\begin{aligned}
x_{\mathrm{Ei}} & =\frac{a_{i}^{n}+S_{T}^{n}}{m_{i}} \frac{r}{S_{T}^{n-1}}\left(1-S_{T} / K\right) ; \\
x_{s i} & =\frac{\left(a_{i}^{n}+S_{T}^{n}\right)^{2}}{m_{i} a_{i}^{n}} \frac{1}{n} \frac{r}{S_{T}^{n-1}}\left(1-2 S_{T} / K\right), \quad i=1,2,3 .
\end{aligned}
$$

Introduce the notation

$$
K_{i}=\frac{\left((n-2) a_{i}^{n}-2 S_{T}^{n}\right) S_{T}}{(n-1) a_{i}^{n}-S_{T}^{n}}, \quad i=1,2,3 .
$$

By condition (2.10) the denominator and a fortiori the numerator are negative, hence $K_{i}$ is positive, further, as the function $f(a)=\frac{\left((n-2) a^{n}-2 S_{T}^{n}\right) S_{T}}{(n-1) a^{n}-S_{T}^{n}}$ is increasing in the interval $a \in\left[0, S_{T} / \sqrt[n]{n-1}\right)$ we have

$$
2 S_{T}<K_{1}<K_{2}<K_{3} .
$$

Now, a simple calculation shows that for $2 S_{T}<K<K_{i}$ we have $x_{\mathrm{Ei}}<x_{s i}$, and $x_{\mathrm{Ei}}=x_{s i}$ when $K=K_{i}$. This implies that for $2 S_{T}<K<K_{1}$ the plane (2.9) is "below" $E$ and reaches $E$ at $x_{E 1}=x_{s 1}$ when $K=K_{1}$. (As $K$ is increased both planes are displaced parallelly). This implies that for $2 S_{T}<K<K_{1}(2.8)$ holds for every equilibrium in $E$. As $K$ is increased further the plane (2.9) cuts into $E$, reaches $x_{E 2}=x_{s 2}$ at $K=K_{2}$, reaches $x_{E 3}=x_{s 3}$ at $K=K_{3}$ and after that cuts the plane of $E$ outside the positive orthant so that now $E$ will be "below" the plane (2.9). In the process, condition (2.8) holds with an inverted inequality sign in the part of $E$ which is already below the plane (2.9). This means that the equilibria on this part of the plane have a two dimensional unstable manifold which fill a neighbourhood of this part of $E$. This proves the Theorem.

Note that $K_{i}$ is positive also if $S_{T} \sqrt[n]{2 /(n-2)}<a_{1}<a_{2}<a_{3}, n>2$, however, in this case the plane (2.9) never reaches $E$. 
Note also that in case of the classical Holling III functional response, i. e., $n=2$ condition (2.10) is just $a_{1}<a_{2}<a_{3}<S_{T}$, the half saturation constants must be less than the threshold value of prey abundance. This means that predator birth rate half the maximal is not sufficient for predator survival. In case of the generalized Holling III functional response the half saturation constants must be even less in order to experience velcro bifurcation.

\section{Computer Images}

In this Section a numerical example will be presented. The classical Holling III functional response will be considered, i. e., $n=2$. The following parameter values are chosen:

$$
\begin{gathered}
r=1, m_{1}=5, m_{2}=6, m_{3}=7, a_{1}=0.5<a_{2}=\sqrt{0.5}<a_{3}=\sqrt{0.75} \\
d_{1}=d_{2}=d_{3}=4 .
\end{gathered}
$$

As a consequence

$$
\beta_{1}=1, \beta_{2}=2, \beta_{3}=3, b_{1}=5 / 4<b_{2}=6 / 4<b_{3}=7 / 4, \quad S_{T}=1 .
$$

Conditions (2.2)-(2.4) and (2.10) hold and the system is

$$
\begin{gathered}
\dot{S}=S(1-S / K)-5 x_{1} S^{2} /\left(0.25+S^{2}\right)-6 x_{2} S^{2} /\left(0.5+S^{2}\right) \\
\quad-7 x_{3} S^{2} /\left(0.75+S^{2}\right), \\
\dot{x}_{1}=\frac{S^{2}-1}{0.25+S^{2}} x_{1}, \quad \dot{x}_{2}=2 \frac{S^{2}-1}{0.5+S^{2}} x_{2}, \quad \dot{x}_{3}=3 \frac{S^{2}-1}{0.75+S^{2}} x_{3} . \\
E=\left\{\left(S, x_{1}, x_{2}, x_{3}\right) \in \mathbb{R}_{+}^{4}: S=1, x_{1}+x_{2}+x_{3}=0.25(1-1 / K), x_{i} \geq 0\right\} .
\end{gathered}
$$

The equation of the plane (2.9) is now

$$
x_{1} / 0.375+x_{2} / 0.225+x_{3} / 0.175=1 .
$$

The critical values of $K$ are: $K_{1}=2.667, K_{2}=4, K_{3}=8$. Condition (2.11) is satisfied. We choose $K=5$. By this, clearly, the points of the plane of $E$ on the axes $x_{1}$ and $x_{2}$ have already been destabilized, but the vertex on the axis $x_{3}$ is still stable.

Figure 1 shows the plane of $E$ cut by the plane (3.2). (The figures were produced by Maple V. The coordinates $S, x_{1}, x_{2}, x_{3}$ are denoted on the figures by $x, y, z, w$, respectively).

We choose now two sets of initial values. The first (denoted by A) is near the destabilized part of $E$ (with $x_{3}$ coordinate small). The second (denoted by B) is near the still attractive part of $E$ (with $x_{3}$ coordinate relatively large). The coordinates of (A) are given by $\left(S(0), x_{1}(0), x_{2}(0), x_{3}(0)\right)=(1,0.08,0.08,0.06)$, and the coordinates of (B) are given by $\left(S(0), x_{1}(0), x_{2}(0), x_{3}(0)\right)=(1,0.01,0.05,0.16)$. Figures 2,3 , and 4 show, respectively, the $x_{1}, x_{2}$, and $x_{3}$ coordinates of the solution. As can be seen, the solution initiating at (A) tends to an equilibrium on the still attractive portion of $E$ far 


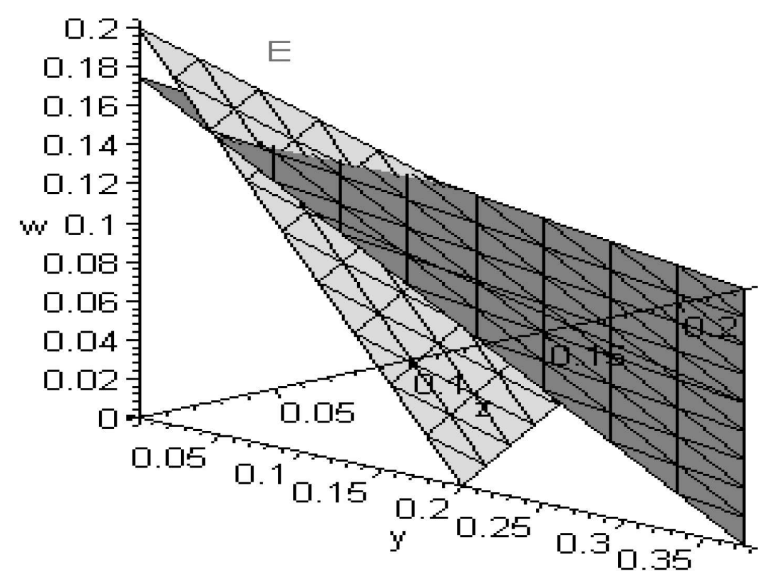

FIGURE 1. Plane of equilibria partly destabilized

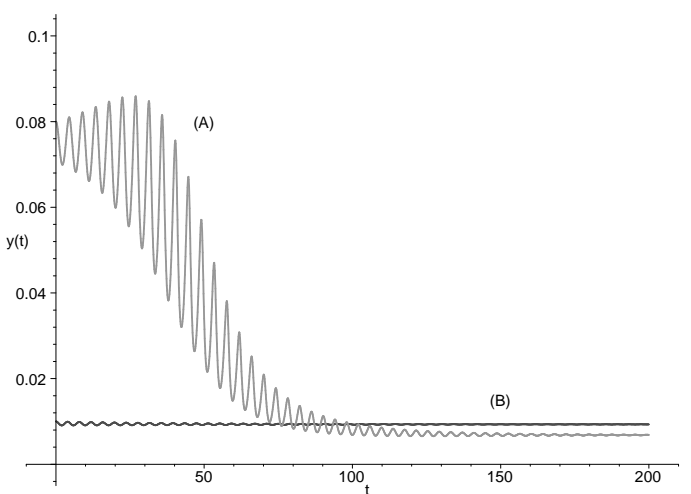

FiguRE 2. $x_{1}$ coordinates of solutions (A) and (B)

from (A) after exhibiting strong oscillations. In contrast, the solution corresponding to (B) tends to an equilibrium on the still attractive portion of $E$ near (B).

Figure 3 shows the $x_{2}=z$ coordinate of these solutions. The behaviour is similar to the previous one.

Figure 4 shows the $x_{3}=w$ coordinate of these solutions. For solution (A) it is increasing considerably, for solution (B) it remains near the initial value.

It is an interesting question what the solutions do when $K>K_{3}$, i. e., when already all the equilibria in $\mathbb{R}_{+}^{4}$ have been destabilized. It is easy to see that the equilibrium point $\left(S_{T}, 0,0, x_{\mathrm{Ei}}\right)$ which is the "last stable one" undergoes a degenerate AndronovHopf bifurcation as $K$ is increased through $K_{3}$. The fundamental Andronov-Hopf bifurcation theorem (see, e. g., [4]) does not apply since at the critical value $K_{3}$ of 


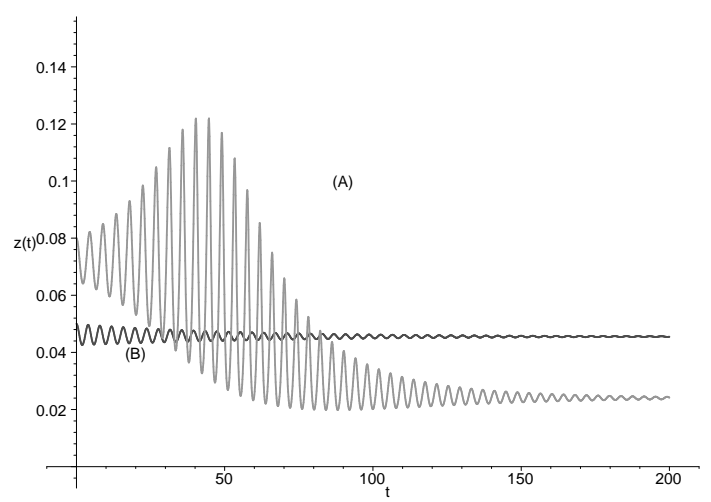

FiguRE 3. $x_{2}$ coordinates of solutions (A) and (B)

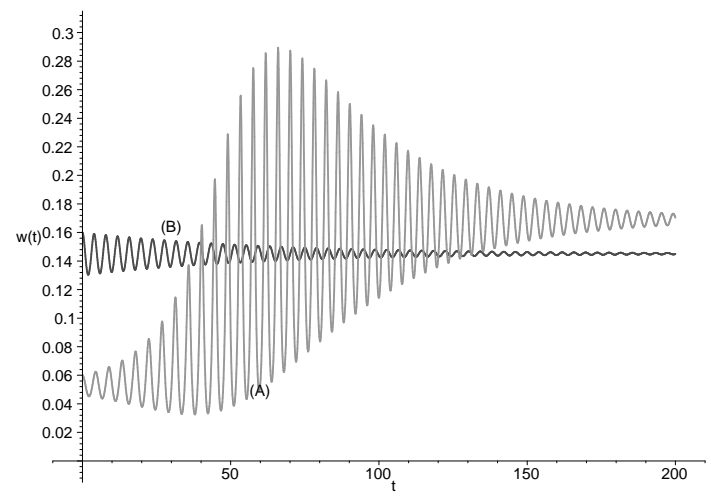

FiguRE 4. $x_{3}$ coordinates of solutions (A) and (B)

the bifurcation parameter this equilibrium has a double zero eigenvalue and a pair of imaginary eigenvalues. Computer simulations show that the closed orbit of the bifurcating periodic solution attracts solutions initiating in $\mathbb{R}_{+}^{4} \backslash E$.

\section{Conclusions}

Three predator species compete for a single prey species. The prey species follows logistic dynamics with carrying capacity $K$ in the absence of predation. The functional responses of the predators are of generalized Holling III type, i. e., increase of prey increases predator efficiency for relatively low prey abundance. All the three predators begin to grow at the same threshold quantity of prey denoted $S_{T}$. Predator 2 is more an $r$-strategist and less a $K$-strategist than predator 1, predator 3 is more an $r$-strategist and less a $K$-strategist than predator 2. We distinguish three values of $K$, denoted herein as $S_{T}<K_{1}<K_{2}<K_{3}$. For $K<S_{T}$, there are no equilibria corresponding to the survival of the predators. For $K>S_{T}$ the system has 


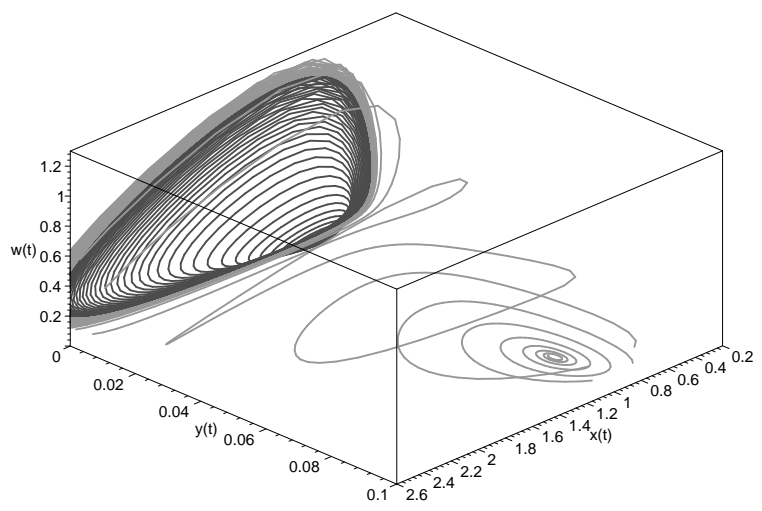

FigurE 5. $K=11$, trajectories tending to limit cycle in the $(x, w)$ plane

an attracting simplex of equilibria in the positive orthant of the 4-dimensional space. This means that solutions tend to a point on this simplex determined by the initial conditions, i. e., the three predator species may coexist with the prey in the long run. Naturally, if neglected and stochastic effects are taken into consideration the limit point may drift on the simplex and this may lead to the extinction of one or two of the predators. As $K$ is increased beyond $K_{1}$, the equilibria on the simplex begin to lose their stability starting with those that represent dominance of predator 1 , the $K$ strategist, and then proceeding on with those that represent dominance of predator 2 . This part of the simplex becomes a repeller of the system and all solutions tend to an equilibrium that represents dominance of predator 3, the $r$-strategist. For values of $K$ larger than $K_{3}$ the first two predators disappear and only predator 3, the $r$-strategist survives with the prey. This is the phenomenon that we call a velcro bifurcation. The results support the intuitively expected fact that abundance of food is advantageous for $r$-strategists, and under such conditions a $K$-strategist loses because its positive properties are of no use. However, if the parameters of the system can be estimated with some exactness, the model tells us at what value of the carrying capacity the $K$ strategist and also the second predator begins to lose ground and at what value they disappear.

\section{ACKNOWLEDGEMENT}

The first author wishes to thank for the hospitality during his stay at the Department for Mathematics of the University Santa Maria of Chile and also thanks Fondecyt-Chile, Grant No. 7030005, for their financial support.

\section{REFERENCES}

[1] Bocso, A., And Farkas, M.: Political and economic rationality leads to velcro bifurcation, Applied Mathematics and Computation, 140 (2003), 381-389.

[2] FARKAS, M.: Zip bifurcation in a competition model, Nonlin. Analysis TMA, 8 (1984), 1295-1309. 
[3] Farkas, M.: Competitive exclusion by zip bifurcation, In: Dynamical Systems, IIASA Workshop 1985 Sopron, Lecture Notes in Economics and Mathematical Systems 287, Springer, 1987, pp. $165-178$.

[4] Farkas, M.: Periodic Motions, Springer-Verlag, New York, 1994.

[5] Gannersdorfer, A., Hofbauer, J., and Sigmund, I.: On the dynamics of asymmetric games, Theoret. Population Biology, 39 (1991), 345-357.

[6] May, R. M. (ED.): Theoretical Ecology, 2nd ed., Blackwell, Oxford, 1981.

\section{Authors' addresses}

\section{Farkas:}

Institute of Mathematics, Budapest University of Technology, Budapest, Hungary, H-1521

E-mail address: fm@math.bme.hu

\section{E. Sáez:}

Departamento de Matemática, Universidad Técnica Federico Santa María, Casilla 110-V, ValPARAISO, ChILE

E-mail address: eduardo.saez@mat.utfsm.cl

\section{Szántó:}

Departamento de Matemática, Universidad Técnica Federico Santa María, Casilla 110-V, ValPARAISO, ChiLE

E-mail address: ivan.szanto@mat.utfsm.cl 